Felix Wermke, Thorben Wübbenhorst, Beate Meffert

\title{
Interference Avoidance for Two Time-of- Flight Cameras Using Autonomous Optical Synchronization
}

2020 | Conference Paper | Accepted Manuscript (Postprint)

available at https://doi.org/10.18452/23670

Final version published as:

Felix Wermke, Thorben Wübbenhorst, Beate Meffert: Interference Avoidance for Two Time-of-Flight Cameras Using Autonomous Optical Synchronization. In: 2020 6th International Conference on Control, Automation and Robotics (ICCAR). DOI: 10.1109/ICCAR49639.2020.9107975

(c) 2020 IEEE. Personal use of this material is permitted. Permission from IEEE must be obtained for all other uses, in any current or future media, including reprinting/republishing this material for advertising or promotional purposes, creating new collective works, for resale or redistribution to servers or lists, or reuse of any copyrighted component of this work in other works.

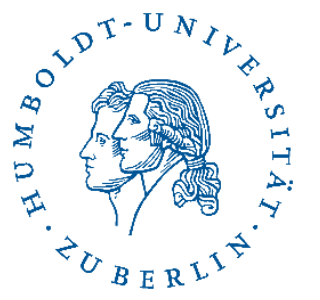

edoc-Server

Open-Access-Publikationsserver

der Humboldt-Universität zu Berlin 


\title{
Interference Avoidance for Two Time-of-Flight Cameras Using Autonomous Optical Synchronization
}

\author{
Felix Wermke, Thorben Wübbenhorst, Beate Meffert \\ Department of Computer Science \\ Humboldt-Universität zu Berlin \\ Berlin, Germany \\ Felix.Wermke@informatik.hu-berlin.de
}

\begin{abstract}
Time-of-Flight (ToF) cameras acquire 3-D images where each pixel relates to the distance in the observed scene. These images are acquired by emitting modulated light and measuring the phase of its reflection from an object. Improvements in ToF camera technology make it a promising alternative to current 3-D imaging sensors despite possessing comparatively lower resolution. Overcoming lower resolution, of single ToF camera images, is achieved using a multi-camera setup. However, the simultaneous operation of multiple ToF cameras causes interference resulting in erroneous depth measurements. An option to mitigate interference is the arrangement of multiple cameras in a consecutive order sharing the same frequency in assigned time slots, such that each camera illuminates the scene exclusively, a procedure known as time division multiple access (TDMA). The assignment of acquisition times is usually performed by a master synchronizing device implementing a time synchronization protocol that requires an additional communication channel. This paper presents a different approach requiring no additional infrastructure to utilize TDMA by adding a synchronization software procedure to the camera's microprocessor which effectively enables a camera to sense the acquisition process of other cameras and rapidly synchronize itself autonomously to operate without interference. Experimental results with two ToF cameras provide substantive evidence of the efficacy of this approach. The operation of the standalone cameras achieved the desired synchronization without illumination interference. The proposed synchronization procedure takes advantages of the zero interference of TDMA and overcomes traditional hard-wired synchronization setups, offering an autonomous and potentially mobile operation of ToF camera deployment.
\end{abstract}

Index Terms-Autonomous synchronization, multi-camera interference, multiple camera system, optical synchronization, timeof-flight cameras.

\section{INTRODUCTION}

A 3-D camera enables the measurement of distance (depth) in a scene which provides a real-world model representing the environment within the camera's field-of-view. Each pixel represents the depth to the recorded point in the scene from the camera's point of view. Time-of-Flight (ToF) cameras capture depth images by emitting modulated infrared light (illumination) and measuring the phase of the reflected light.

*This research was performed in cooperation with iris $\mathrm{GmbH}$ and is cofinanced by the European Regional Development Fund (ERDF)
Improvements in ToF camera technology make it a promising alternative to current 3-D imaging sensors and have expanded the field of 3-D imaging applications.

An evolving application is collaborative robotics. Collaborative robots, the cobots, work with humans either directly or in a shared work space environment. To minimize the risk of physical contact between humans and cobots, additional necessary safety precautions must be undertaken. A 3-D representation of the robotic environment is necessary which can be utilized by ToF cameras [1]-[4]. However, a major disadvantage of commercially available ToF cameras is their lower image resolution (in the order of 320x240 pixels) compared to other 3-D imaging systems like stereo vision cameras. A lower resolution may be acceptable in smaller defined areas but not in larger industrial environments. The fusion of multiple depth images from a multi-view ToF camera system enhances the 3-D representation of the environment by increasing the spatial resolution, covering more of the scene, and reducing occlusion (shadowed objects unable to be seen from particular fields of view). Multiple ToF cameras, operating simultaneously, create a resultant light waveform interference during their image capture phase. This interference leads to errors in depth computations. Therefore, light interference reduction strategies are necessary when using multi-ToF-camera setups [5]-[9]. One option is to reduce interference artifacts in post-processing by statistical calculations [8], [10]. Preferably, interference is already reduced during image acquisition by applying channel access methods. A channel access method defines how the transmission medium is shared between multiple cameras. The most commonly used method is frequency division multiple access (FDMA), where each camera is assigned a unique modulation frequency for the illumination. Other access methods include the use of different wavelengths of the infrared spectrum (WDMA), the application of codes to the illumination (CDMA), or assigning time slots to the same modulation frequency, where each camera is assigned exclusive image acquisition times (TDMA). An overview of channel access methods may be found in [11], [12] with their respective advantages and disadvantages. A 
short overview is provided below. FDMA employs independent camera operation using different modulation frequencies to minimize (but not completely eliminate) interference artifacts. Code division multiple access (CDMA) was developed to overcome the shortcomings of FDMA through the use of orthogonal modulation signals to mitigate interference, albeit requiring more sophisticated hardware. The time division multiple access (TDMA) method avoids the limitations of camerainduced interference by assigning exclusive consecutive time slots for image acquisition. However, the introduction of time slots reduces the maximum frame rate per camera because of the longer pauses between image acquisitions per camera. Additionally, these setups are usually static and operationally more complicated, i.e. a predefined number of cameras is hardwired to a master synchronizing or coordinating device that provides time synchronization and assigns the cameras to time slots.

A method to overcome the limitation of a static setup of TDMA was proposed by Wübbenhorst [13]. He successfully tested a synchronization procedure with two ToF cameras operating without wiring the cameras or the necessity of an external master synchronizing device. Wübbenhorst proposed using additional photodiodes attached to the cameras which will detect the completion of the acquisition time of the other camera and trigger its own acquisition phase.

This paper proposes a new methodology to operate multiple ToF cameras using time division multiple access (TDMA) for creating 3-D representations of the environment. It operates without interference, minimal reduction in frame rates, and none of the traditional TDMA mobility limitations, negating the need for an external synchronizing device requiring hardwired setups, thereby achieving a dynamic and expansive autonomous camera operation. It can be applied to existing systems without modifying camera hardware. The basis of this work is an autonomous assignment of time slots by each camera individually. The software in the camera's microprocessor is extended by the development of an additional synchronization procedure. In addition to the normal image acquisition operation, an optical synchronization algorithm is performed. It enables the camera to sense another camera's image acquisition process and adapt its own image acquisition times autonomously.

Frame rate reduction associated with increasing numbers of operating cameras is addressed by interleaving operational cycles. After a camera's integration phase with illumination, pixel data is transferred from the image sensor to the microprocessor (the readout phase), resulting in a time interval for the camera with no illumination. Other cameras can now begin their image capture phase consecutively in the preceding camera's readout phase time interval without interference.

Because of its autonomous operation, the system can be viewed as a self-configuring system suited for flexible or mobile environments. The synchronization procedure proposed will allow dynamic and flexible real-time robotic activity, allowing for multiple and dynamically changing ToF camera operation without any modification of environmental setup.

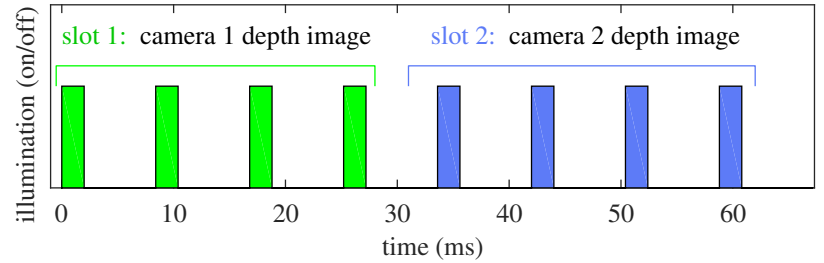

Fig. 1. Image acquisition of two ToF cameras. One depth image per camera is acquired consecutively, each consisting of 4 frames. Implementing TDMA, each camera is assigned to a time slot for each single depth image acquisition process. The length of the time slot is the required time for a single depth image acquisition. The bars represent the active illumination during frame acquisition. Between the illuminations/bars the readout process takes place.

For example, moving robots with mounted ToF cameras for collision avoidance may enter new environments with multiple cobots/humans adapting themselves to the new situation.

This paper presents research for an initial proof of concept using two ToF cameras but the proposed synchronization algorithm is nevertheless applicable to multiple $\mathrm{ToF}$ camera environments.

This paper is organized as follows. Section II explains the operating principle of ToF cameras and illustrates the effect of interference. The autonomous synchronization algorithm developed is explained in Section III. The experimental setup is presented in Section IV, the measurements and results are provided in Section V. The article closes with discussions in Section VI and conclusions in Section VII.

\section{OPERATING PRINCIPLE OF TOF CAMERA}

A ToF camera consists of an image sensor, an illumination unit, and a microcontroller to orchestrate the operation. Amplitude modulated continuous wave (AMCW) ToF cameras emit amplitude modulated light (illumination), which is reflected by objects in the scene, and then recorded by the camera's image sensor. This light's round-trip time is proportional to the distance to the object and is determined indirectly by measuring the phase of the reflected, usually sinusoidal-modulated, wave instead of the actual round-trip time. To determine the phase shift, the reflected lightwave is sampled four times, at $0^{\circ}$, $90^{\circ}, 180^{\circ}$, and $270^{\circ}$ of the carrier's wavelength. The distance (depth) is then calculated from the recovered phase. The details are explained in Seitz and Lange [14].

A ToF camera acquires multiple samples (frames) to record a scene. Frame acquisition involves the following primary stages - image capture (shutter, illumination, and integration) and readout (digitization and data signalling). The microcontroller starts the frame acquisition process by activating the image acquisition trigger signal. When commencing a frame acquisition, the illumination unit is turned on (illuminating the scene), the shutter is opened, and the pixels are then light sensitive (integration phase). When the set integration time has passed, the illumination is turned off, and the shutter closed. Illumination refers to the emission of light whereas the integration refers to the process of receiving light in the image sensor when the shutter is open. The integration phase is 

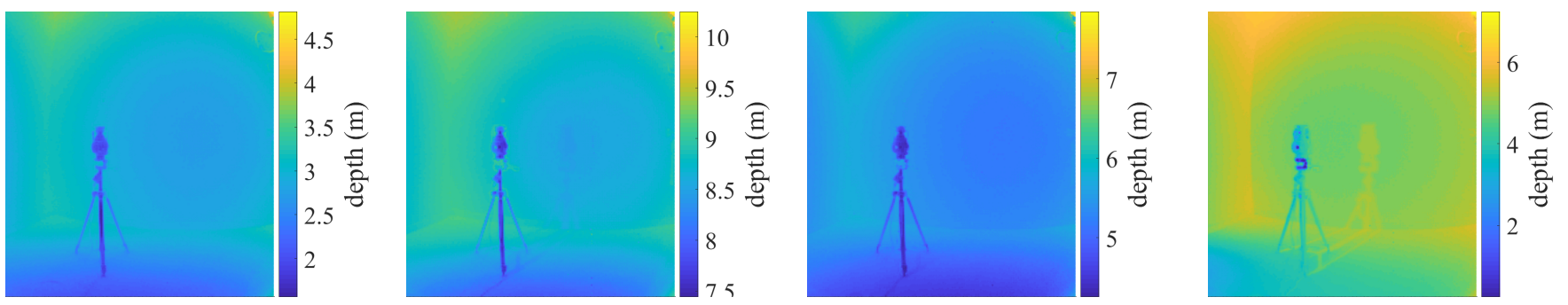

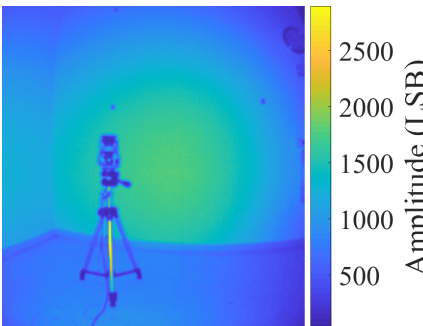

(a) Only one camera $(\mathrm{d}=1.9 \mathrm{~m})$

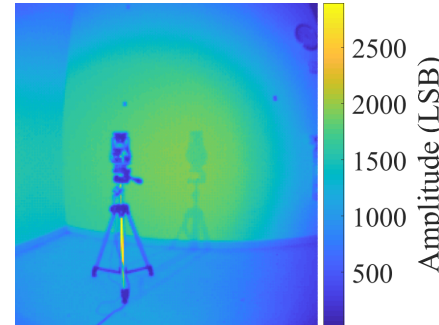

(b) $\phi=0, \mathrm{~d}=7.6 \mathrm{~m}$

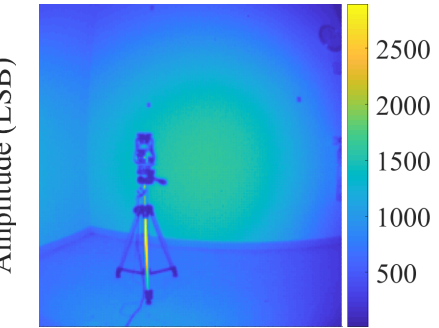

(c) $\phi=27 \mathrm{~ns}, \mathrm{~d}=4.3 \mathrm{~m}$

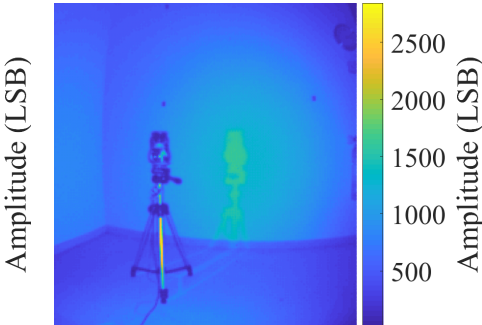

(d) $\phi=30 \mathrm{~ns}, \mathrm{~d}=3.9 \mathrm{~m}$

Fig. 2. Images to illustrate the erroneous depth calculations due to interference of cameras' illuminations. The scene is constant but the measured distance d to the object varies due to interference. Image (a) without any interference. Images (b), (c), and (d) represent interference effects with different phase shifts $\phi$ between the illumination of two simultaneous operating ToF cameras. Top row shows the depth image, bottom row the amplitude image. Please note the differing scales between depth images.

followed by the readout phase, where the pixel data is digitized and transferred from the image sensor to the microcontroller. The microcontroller stores the frames and calculates the depth images afterwards. As the standard operational design of a ToF camera samples the amplitude of reflected light four times to determine the phase four frames are required for a single depth image. The distinction between a depth image and the four required frames for its calculation is important. Fig. 1 shows the consecutive frame acquisition of two ToF cameras. Two depth images consisting of four frames each are acquired. The bars represent the active illumination/integration.

When two light waves superpose they form a different wave. This phenomenon is called interference. When using more than one camera, it is a known problem that interference occurs when their illumination units are simultaneously active. The recorded sample value of the reflected light is modified by interference. To illustrate the interference effects, a series of images is shown in Fig. 2. The images in the same column show the depth image (top) and amplitude of the received illumination (bottom) of the same image acquisition. The left image (a) displays the reference scene, without any interference. Images (b), (c), and (d) represent interference effects with different phase shifts between the illuminations of two simultaneous operating ToF cameras. The phase shift between the illuminations of the two cameras was varied. The actual phase $\phi$ is provided in the image caption, together with the measured depth $d$ of the object. It can be seen that the calculated depths are erroneous and vary depending on $\phi$. Interference should be avoided to achieve correct depth measurements.

\section{PROPOSED SYNCHRONIZATION ALGORITHM}

Using time division multiple access (TDMA), a channel access method to define exclusive acquisition times by providing time slots, requires a synchronizing unit, usually implemented by wiring the participating devices. Replacing this additional wired complexity, research was undertaken to develop a software algorithm to manipulate the existing microcontroller's firmware to create a self-contained synchronizing camera, in addition to its default image acquisition operation.

The procedure in its simplest form is turning on the cameras one after another and the synchronization algorithm in each camera autonomously determines timings of operation. The first step after power on is to detect other operating cameras. The first operational camera searches for illumination from another camera, finds none, and operates independently and unimpeded, therefore called the "free-running" camera. On power up, each subsequent camera detects other operating cameras and commences operation by initially executing the optical synchronization algorithm, hence referenced as "synchronizing" camera.

The optical synchronization algorithm enables the cameras to operate in a synchronous manner. This requires two steps. First, the camera has to detect the presence of another camera operating nearby by sensing modulated light of other cameras. At the same time, also the number of already operating cameras needs to be determined. Second, the synchronizing camera has to determine the free-running camera's illumination interval, with respect to its own frame acquisition cycle. Then, the own frame acquisition cycle has to be shifted to operate within an unused time slot without interference. The proposed synchronization algorithm cannot detect the size of 
the required time shift at once but rather uses an iterative process to align itself to the time slot. Fig. 3 shows a flow diagram of the operation procedure of a ToF camera with the additional synchronization procedure.

There are distinct operating procedures between 'normal' mode, which refers to the depth image acquisition of cameras, and 'optical synchronization' mode. The normal mode, acquiring depth images, is active most of the time. It can be paused to run the synchronization. Due to hardware and environmental differences, e.g. different temperatures at the cameras, drift in timing will occur for all cameras and the acquisition trigger times within the time slot will deviate. To ensure a synchronized state (operation without interference), the synchronization algorithm initially and periodically calculates the correct image acquisition trigger point for the commencement of its frame capture and adjusts its trigger accordingly by slight time shifts.

The conceptual development of the synchronization procedure was envisaged for a multiple-camera operation. As this paper discusses the mandatory first stage research for two autonomously operating ToF cameras, any reference to a multiple-camera operation is limited and outside the scope of this paper.

For theoretical and practical considerations of achieving a high sensing accuracy it is necessary that the free-running camera uses a constant illumination time. The discussion section addresses the rationale for this requirement and the feasibility of adoption of the procedure for real-world applications.

Subsection III-A explains the implementation of TDMA. Subsection III-B describes the detection mode of another operating camera and subsection III-C explains the algorithm's determination of the time slots and the mechanics of timeshifting procedure. The algorithm is performed continually at user-defined intervals to maintain a synchronized state. Subsection III-D provides more details about this synchronization interval. The error of the synchronization procedure is covered in subsection III-E.

\section{A. Time division multiple access}

Time division multiple access (TDMA) is a channel access method for a shared transmission medium. Assigning exclusive image acquisition times (time slots) to multiple cameras, operating on the same frequency, is a method to avoid the limitations of camera-induced illumination interference. However, the introduction of consecutive time slots reduces each camera's maximum frame rate due to delaying or waiting for each camera's dedicated time slot.

The simplest form of TDMA may be described operationally as one camera sequentially performing an image acquisition cycle requiring four frames for depth image calculations in its assigned time slot, then waiting for the next camera to complete its image acquisition cycle before resuming operation. This results in a frame rate reduction. Fig. 1 shows the consecutive image acquisition of two ToF cameras.

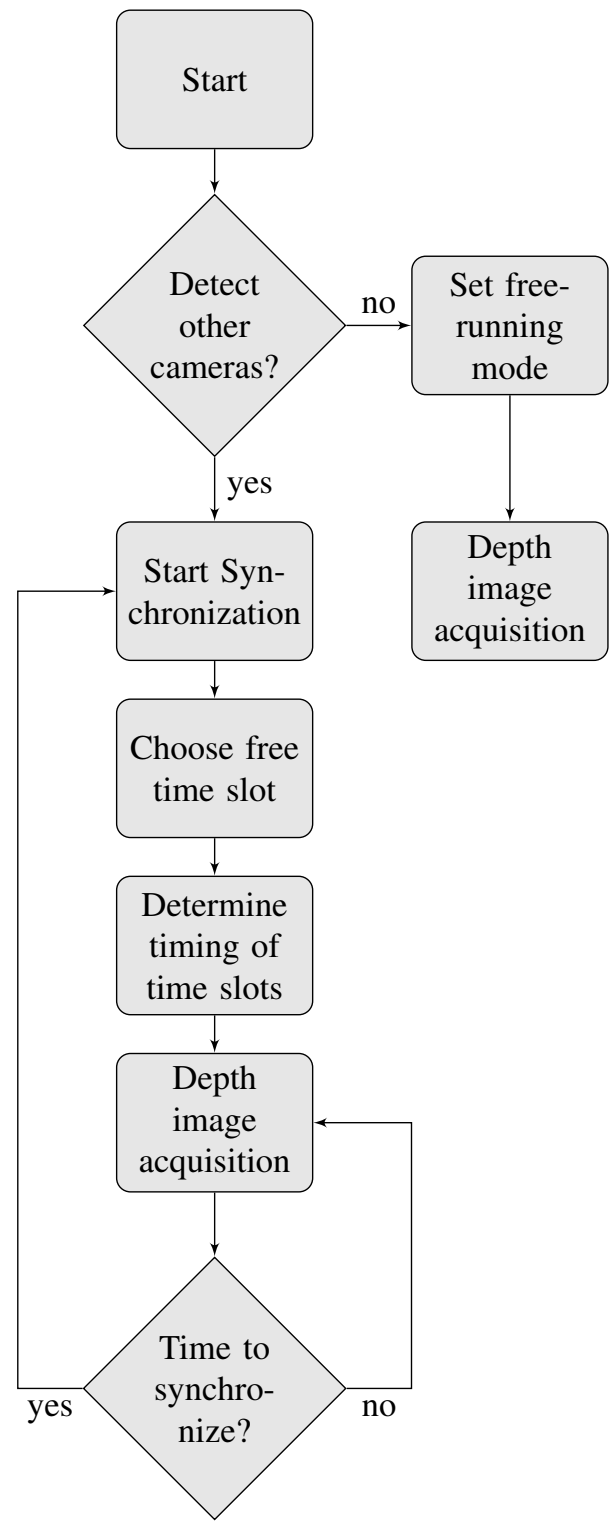

Fig. 3. Flow diagram of camera operation. Operation is extended with the synchronization procedure.

During each depth image acquisition, the integration phase (in order of $\mu \mathrm{s}$ to $\mathrm{ms}$ ) and the longer readout phase (in $\mathrm{ms}$ ) are performed four times. As there is no illumination during the readout phase, it is an inefficient use of channel access time as the next camera waits for the previous camera to complete its cyclical operation of image capture and then commences operation in its assigned TDMA time slot.

To exploit this inefficient use of the transmission medium and frame rate reduction, a different implementation of TDMA is proposed. Essentially, the multi-camera sequential image acquisition is replaced with an interleaved time-shifted synchronous operation. Multiple cameras may synchronize to operate freely without waiting times immediately after the illumination of the previous camera's integration phase, exploiting the non-illuminating readout phase of the previous camera. 

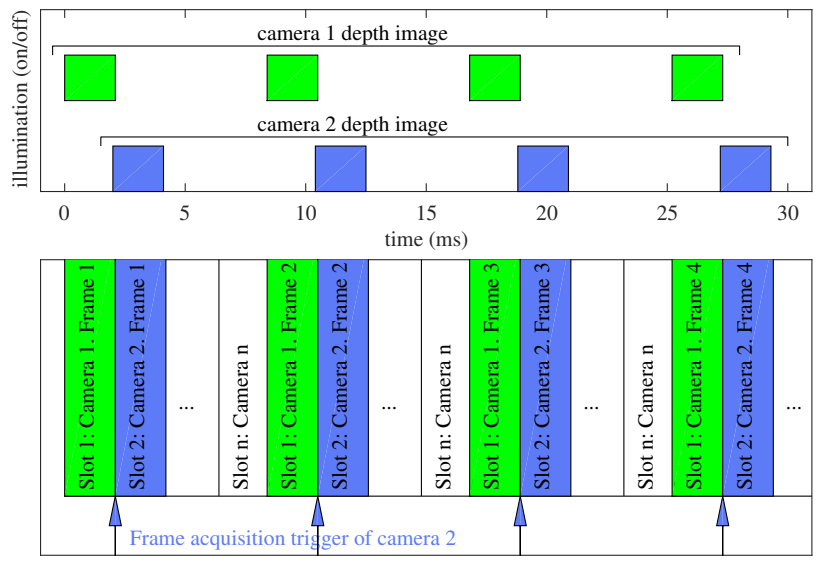

Fig. 4. Graphical representation of time slots of TDMA. Two cameras (green and blue) are assigned to time slots, where only the assigned camera is allowed to illuminate the scene. The duration of the time slot covers the integration phase of one frame. The time slots repeat after slot $n$. The time required to acquire two depth images is shorter compared to the TDMA implementation in Fig. 1. The frame acquisition triggers of camera two are indicated by the arrows at the bottom.

Fig. 4 shows the arrangement of time slots with the short time slots of TDMA. Every time slot allows the assigned camera (green and blue) to run the integration phase (illumination of the scene). The frame acquisition trigger of camera 2 is illustrated by a blue arrow at the bottom of the figure. The readout process of the frame follows after the assigned time slot.

The duration of one frame acquisition (integration + readout time) is divided into a predefined number of slots $n$, depending on the readout time $t_{\text {readout }}$, the maximum integration time $t_{i n t}$, and the maximum number of operating cameras. The slot length has to be greater than the maximum integration time. Therefore the maximum number of time slots is floor $\left(t_{\text {readout }} / t_{\text {int }}\right)$. These slots are consecutive in time and repeat after every $n$-th slot. The free-running camera is assigned to slot 1 as it dictates the timing of the time slots. Each synchronizing camera is assigned to a slot number $2 \ldots n$ and illuminates the scene only when its slot is active, limiting the integration time to the slot length. The readout process of each synchronizing camera takes place during the following $n-1$ time slots. Using this interleaving method, multiple cameras may operate with near negligible frame rate loss.

\section{B. Detection of other cameras and slot assignment}

The first step after power on is to detect other operating cameras. This is performed by acquiring a frame with no illumination and extended integration time to cover all time slots. The first operational camera searches for illumination from another camera, finds none, and is assigned to the first TDMA time slot. The algorithm sets the camera into 'freerunning operation'. That is, the logic directs it to free-running mode and no further synchronization routine. The free-running camera dictates the timing cycles for the TDMA time slots. This definition of time slots and the relation of the cameras to the time slots must be emphasized. There is no central synchronizing device and the cameras operate autonomously without communication between each other. This means that there is no common reference time between the cameras. By the predefined parameters of TDMA, the number and duration of time slots are known but not the starting time. The freerunning camera, assigned to the first slot, defines the start of the first frame by illuminating the scene. The synchronizing cameras have to detect that illumination and infer the timing of the first time slot. Then they can determine the timing of the subsequent time slots and assign themselves to the next available time slot.

In mobile environments, e.g. when the ToF cameras are mounted on a robot, the detection of other possibly operating cameras needs to be executed multiple times. This detection may be triggered by the robot based on its movement.

\section{Determination of timing of time slots}

If the free-running camera is detected, the timing of the illumination period of the free-running camera must be determined. The free-running camera defines the time slots and the synchronizing camera has to operate within a selected time slot. The synchronizing camera has to detect when the free-running camera's illumination starts and stops in order to determine the timing of the time slots. Therefore, a precise time measurement is necessary, which requires a high temporal resolution and therefore small integration times as the duration of the integration time represents the granularity of the time resolution. Simply reducing the integration time and acquiring a lot of frames with a high frame rate is not possible because every integration phase is followed by the readout phase. During the readout phase the presence of illumination cannot be detected. Furthermore, small integration times lead to low signal-to-noise ratios due to sensor noise [15], thus decreasing the capability to accurately determine the presence of modulated light. To overcome the problems of a low temporal resolution, sensor noise, and long readout times, the optical synchronization algorithm was developed introducing a new mode of operation for the synchronizing camera, named 'finesync mode'. This mode operates with different acquisition parameters compared to the camera's normal depth image acquisition and is only used to retrieve timing information about the time slots. While the fine-sync mode operates, the normal mode pauses. When the synchronization procedure is finished, the normal mode continues to acquire depth images.

In fine-sync mode, two frames (sync-frames) are acquired in a time interval of one 'normal' frame acquisition. These two sync-frames are acquired with specific timing parameters, such that both their integration times fit into the normal mode's readout time, covering $n-1$ time slots. In the synchronized state, the first sync-frame's integration phase starts immediately after the completion of the illumination phase of the free-running camera and the second sync-frame's integration phase ends just before the next commencement of free-running camera's illumination phase of the next cycle. Thus, the system is considered synchronized if both of these sync-frames receive no illumination. Using the described timing of the sync-frames 


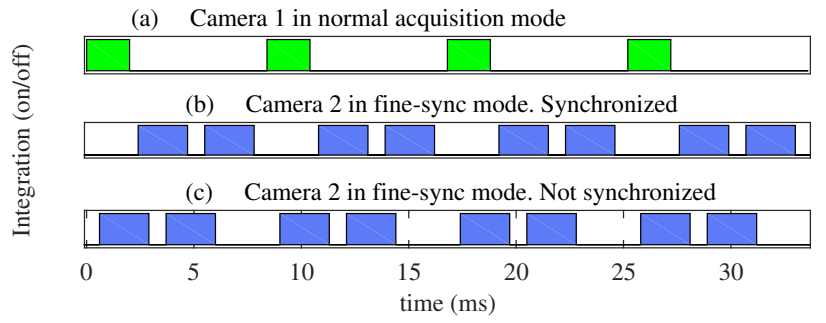

Fig. 5. Two cameras in different acquisition modes. The bars indicate the active integration times. (a) shows the normal acquisition mode of the freerunning camera to acquire depth images. (b) and (c) show the fine-sync mode of the synchronizing camera. (b) shows the synchronized state and (c) a nonsynchronized state.

allows the determination of the timing of the free-running camera's illumination phase. In the non-synchronized state, one of the two sync-frame's integration phase overlaps with the free-running camera's illumination phase and therefore receives illumination.

Fig. 5 shows the normal acquisition mode and the finesync mode. When operating in the normal mode (a), depth images are acquired with active illumination. (b) and (c) illustrate the fine-sync mode with the two sync-frames in the synchronized and non-synchronized state. In fine-sync mode no light is emitted, therefore the blue bars represent active integration time. If the two cameras are not synchronized ((a) and (c)), one sync-frame of the fine-sync mode is illuminated by the free-running camera. In (b), the integration times of the sync-frames do not overlap with the illumination of the freerunning camera. These two sync-frames fit into the readout time of the free-running camera and therefore do not record any illumination.

The non-synchronous state is determined, when either of the sync-frames receives illumination. As the recorded illumination amplitude also consists of noise, it is difficult to distinguish between low illumination and noise. The problem can be solved by comparing the two sync-frames. Since there is only one illuminated sync-frame, the other serves as a reference for the recorded noise. The amplitudes of all pixels are summed up for comparing the sync-frames' amplitudes. Due to the noise, these amplitudes cannot be simply compared. A threshold was determined empirically to be twice the amplitude of all pixels' sum compared to the other sync-frame to distinguish the illuminated sync-frame from noise.

When the threshold is exceeded, one sync-frame is considered to have received illumination. For two sync-frames, recording just noise and no illumination, twice the amplitude of noise is still statistically insignificant, compared to syncframes with actual illumination. However, in order to exceed the threshold, some overlap of the free-running camera's illumination with one of the sync-frames is required, this time is named 'overlap time' $t_{\text {overlap }}$. Whilst detecting overlapping illumination, the software cannot determine the amount of overlap from the comparative sync-frames, because the received light amplitude depends on the scene. The software proceeds with the iterative process of time-shifting the acquisition trigger point to find the correct end of the illumination phase of the free-running camera. After time-shifting the trigger, the fine-sync mode starts over until the state is synchronized.

The time shift of the synchronizing camera's image acquisition trigger is performed by the optical synchronization control algorithm in the microcontroller. It is possible to shift the acquisition time such that both sync-frames do not receive any illumination because the fine-sync mode's timing parameters are defined so that the sync-frames' integration phases fit into the default readout time. Whether the next acquisition time point needs to be delayed or advanced depends on which syncframe is illuminated. If the first sync-frame is illuminated, the next acquisition cycle needs to be delayed and vice versa.

The predefined time shift value must be considered, possibly ranging from very small to large step sizes. A small shift allows for a fine-grained repositioning of the trigger but requires more iterations of the synchronization until the trigger has reached the synchronized point. A larger shift reduces the required number of shifts but in turn cannot shift the trigger such fine-grained. This trade-off must be considered when defining the step size $t_{\text {time_shift }}$. The process of shifting the trigger with large shifts can lead to a situation where the trigger time points are shifted too far, past the point of synchronization, such that the fine-sync mode then detects that the other sync-frame is illuminated compared to the previous iteration. This might lead to an oscillation of shifting back and forth, which could enter a state where it will never end shifting. Possibly smaller time shift step sizes reduce the chance of this oscillation but require more synchronization iterations. To overcome this problem and to reduce the number of iterations and ultimately minimize frame rate reduction of depth images caused by the synchronization procedure, the integration time of the sync-frames is trimmed slightly (in the order of $\mu \mathrm{s}$ ) and this reduction is named 'gap'. This was deemed acceptable in terms of meeting the condition of synchronization using only few iterations in comparison to the synchronization error induced by the gap. The gap size $t_{g a p}$ is preferably larger than the time shift size $t_{\text {time_shift }}$.

For a two camera setup, in fine-sync mode the following equation has to be satisfied:

$$
2 \cdot t_{\text {int_sync }}+t_{\text {readout_sync }}+t_{\text {gap }}=t_{\text {readout }}
$$

The left side of the equation represents the parameters of the fine-sync mode of the synchronizing camera, whilst the right side is the constant readout time of the free-running camera. In the fine-sync mode, the readout $t_{\text {readout_sync }}$ and integration times $t_{\text {int_sync }}$ differ from the normal mode acquisition parameters. Two integration phases and one readout phase need to be accommodated in the default readout time. The readout time of the second sync-frame occurs when the freerunning camera starts its next acquisition cycle and therefore only one readout cycle is considered in the equation. In order to satisfy the equation, the readout time $t_{\text {readout_sync }}$ must be reduced. The readout time may be reduced by several methods such as 'Binning' (accumulating neighboring pixel values and reducing the number of pixels to be read out) or by defining 
a region of interest (only a predefined subsection of image is read out from the image sensor to the microcontroller) because the full image resolution is not required for the synchronization procedure.

Once synchronized, the synchronization procedure shifts the acquisition trigger to the beginning of the assigned TDMA time slot, and changes back to normal operating mode. During the fine-sync mode, the normal mode is paused leading to a reduction of acquired depth images. The duration of the pause is minimum one frame acquisition long, required to determine that the current state is still synchronized. Otherwise, every iteration requires another pause of one frame acquisition duration.

\section{Synchronization interval due to drift}

The ToF camera commences the frame acquisition in its assigned TDMA time slot. The time between two successive frames is constant and equal to one TDMA cycle. The constant frame rate is realized by implementing a timer (counter) in the camera's microprocessor. When the timer reaches a predefined value, the image acquisition trigger is activated, starting an image acquisition, and the timer is reset to zero and continues counting up again. The predefined value and the rate of increments defines the camera's periodic frame acquisition time with all the phases of operation. The time between two increments of the timer is controlled by an oscillator with a known nominal frequency. The frequency stability of an oscillator depends upon its frequency drift caused by component aging and environmental influences (e.g. temperature). This frequency drift, identified in manufacturers specifications, consequentially results in a 'time-based drift' of the synchronizing camera's image acquisition trigger point within the assigned time slot, thus necessitating consideration of a buffer (or guard interval) within proposed TDMA time slots to guarantee illumination times within the assigned time slot.

In practical terms, frequency drift is compensated by determining a buffer size within the time slot and performing the synchronizing procedure periodically to ensure the synchronizing camera's image acquisition trigger point remains within its assigned TDMA slot. The buffer size depends on the trigger's drift during the synchronization interval, the time between two synchronizations. The drift $t_{d r i f t}$, the maximum amount of time shift of the trigger per synchronization interval (here: $\mathrm{s} / \mathrm{s}$ ), requires a buffer size of $2 \cdot t_{\text {drift }}$ because the drift can go in two directions depending on whose camera's oscillator has the higher frequency.

Oscillators manufactured with high frequency stability result in less frequency drift, therefore less drift in camera acquisition trigger points. Consequently, buffer values can be reduced thereby offering the potential for a greater number of TDMA time slots and the potential to increase the number of cameras able to operate during the free-running cameras readout cycle. Alternatively, instead of decreasing the buffer size the synchronization interval can be increased. If, hypothetically, an oscillator was manufactured with no frequency drift, the optical synchronizing algorithm would only be required to be run once with no drift in the synchronizing cameras image acquisition trigger point and no requirement for a buffer within the TDMA slot to prevent interference.

The frequency stability is quantified in the maximum offset to the nominal frequency using the relative unit parts per million (ppm). For example, two oscillators with a frequency stability of $\pm 25 \mathrm{ppm}$ will result in a maximum relative frequency difference of $50 \mathrm{ppm}$. This corresponds to a relative time shift of the acquisitions triggers of $50 \mu \mathrm{s}$ per second. Having a synchronization interval of, for example, $10 \mathrm{~s}$ would require a buffer size of $0.5 \mathrm{~ms}$.

\section{E. Synchronization error and buffer}

Oscillators possess frequency drift necessitating continually performing the optical synchronization algorithm to reset any drift of the acquisition triggers position in its assigned TDMA time slot. The buffer size compensating frequency drift is 2 . $t_{\text {drift }}$.

Furthermore, during the synchronization procedure itself, the position of the sync-frames in regards to the free-running camera cannot be determined without error. This is due to the very basis of the sensing process itself (overlap time) and the controller's parameters (gap size and time shift size) to function without oscillation. When positioning the acquisition trigger into its time slot, the actual position varies due to these synchronization errors requiring an increase of the buffer within the time slot. The overlap time $t_{\text {overlap }}$ is a mandatory time interval (in $\mu \mathrm{s}$ ), required by the sensing process to assess whether the criteria for a synchronized or non-synchronized state is satisfied. A trial of measurements of the scene is required to measure the value as it is not known a-priori. The synchronization error introduced by the controller's parameters (gap size and trigger shift size) in order to operate without undesired oscillations of time shifts is $\max \left(t_{\text {time_shift }}, t_{\text {gap }}\right)$. Avoiding oscillation is achieved by reducing the sync-frames' integration time slightly, introducing a 'gap'. This gap creates a time interval $t_{\text {gap }}$ in which the sync-frames can drift without detecting any illumination. Therefore, the acquisition trigger position may shift, due to drift, within that interval while the state is considered synchronized. The time shift size $t_{\text {time_shift }}$ contributes to the error only in the case that it is larger than the gap size. The maximum total buffer size for every time slot, including the buffer to compensate for drift and synchronization errors, is

$$
t_{\text {buffer }}=2 \cdot \max \left(t_{\text {overlap }}, t_{\text {drift }}\right)+\max \left(t_{\text {time_shift }}, t_{\text {gap }}\right)
$$

In case the direction of the drift does not change, the equation is simplified to:

$$
t_{\text {buffer_constant_drift }}=\max \left(t_{\text {overlap }}, t_{\text {drift }}\right)+t_{\text {time_shift }}
$$




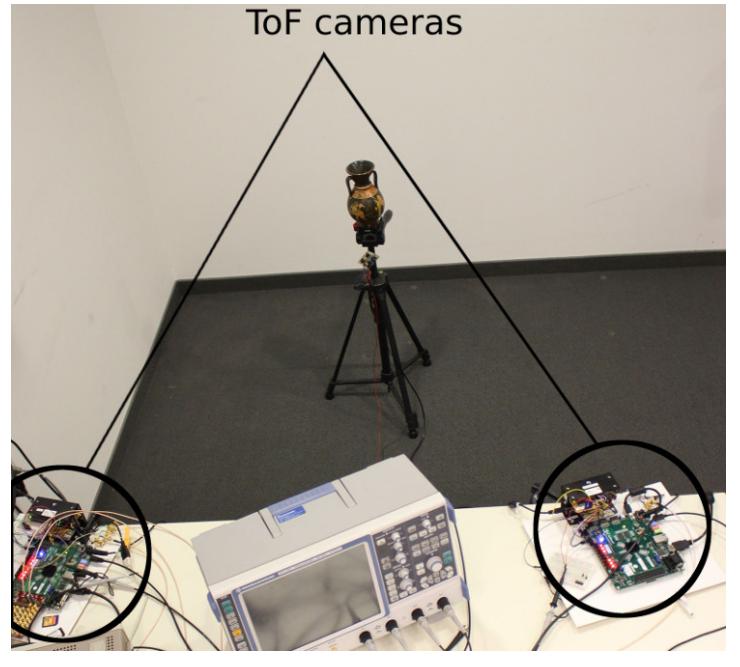

Fig. 6. Experimental setup with two ToF cameras, left and right (Zedboards are green and DME660 black), observing the same scene. Both ToF cameras capture the same object, visible in the middle, from different perspectives A simultaneous image acquisition may lead to interference, and therefore erroneous measurements.

\section{EXPERIMENTAL SETUP}

Experiments were conducted to evaluate the proposed optical synchronization procedure. Fig. 6 shows the experimental setup. It consists of two ToF cameras, observing the same object from different perspectives. The ToF cameras from the evaluation kits DME 660 from ESPROS Photonics Corporation were used for the experiments. Each camera is composed of an image sensor board, an illumination board, and a microcontroller board, which controls the image sensor and illumination, and calculates the 3-D depth image. In this setup, the microcontroller board was replaced by a Zedboard equipped with a Field Programmable Gate Array (FPGA). The FPGA configures the image sensor and stores the acquired frames for depth image calculation and analysis. In addition, the FPGA controls the image acquisition time. A timer, driven by an oscillator with a frequency stability of $\pm 25 \mathrm{ppm}$, is implemented that continuously starts image acquisition using a separate wire, which is connected to the camera's acquisition trigger signal. This signal is referred to as a 'trigger' below and will be used to evaluate the experiments. The time between the triggers indicates whether the illuminations of the two cameras overlap. The FPGA can alter the time between two consecutive image acquisition leading to a shift of the trigger in regards to the time slots.

\section{Measurements And Results}

For the experiments, parameters for image acquisition and synchronization were replicated on both cameras.

The integration time $t_{i n t}$ is fixed to $2 \mathrm{~ms}$. The readout time $t_{\text {readout }}$ for the whole pixel field is $6.4 \mathrm{~ms}$. The parameters for the synchronization procedure are $t_{\text {int_sync }}=2.2 \mathrm{~ms}$, $t_{\text {readout_sync }}=1.7 \mathrm{~ms}$, and $t_{\text {gap }}=0.3 \mathrm{~ms}$. The controller uses a constant time shift value of $t_{\text {time_shift }}=0.2 \mathrm{~ms}$ for shifting the acquisition trigger.
Two measurement series were conducted to evaluate the synchronization procedure. The first series was undertaken to measure the synchronization error of the proposed optical synchronization procedure with the experimental setup. Both cameras operated independently, using optical synchronization only. One camera operated in free-running mode during the test whereas the second camera synchronized itself to the former. After every 299 images recorded, $\approx 10 \mathrm{~s}$, a synchronization procedure was automatically initiated. The synchronization test was performed for $60 \mathrm{~min}$ and approximately 100000 trigger samples were recorded. Whenever the microprocessor of the free-running camera activated the trigger to acquire an image, the time difference between the two cameras' triggers is recorded. That time difference corresponds to the position of the synchronizing camera's acquisition trigger within its time slot. The actual measured frequency difference of the independently running oscillators was $45 \mathrm{~Hz}$ at $12 \mathrm{MHz}$ (3.75 ppm), which corresponds to a time drift of $t_{\text {drift }}=0.0375 \mathrm{~ms}$ every $10 \mathrm{~s}$. For this setup, although it was not recorded, it was expected that the drift did not change its direction during the test. The expected maximum buffer size accommodating all triggers' position, calculated using (3), is minimum $0.2375 \mathrm{~ms}$ as $t_{\text {overlap }}$ is unknown. The measurement was performed using an oscilloscope, which also provided the statistics. Fig. 7 shows the histogram and TABLE I shows the characteristic values of the histogram. In $99 \%$ of the acquired images the positions of the trigger of the synchronizing camera fall into a window of $\pm 2 \cdot \sigma= \pm 0.12 \mathrm{~ms}$. Most histogram samples are within the range of $\approx 0.2 \mathrm{~ms}$, which corresponds to the trigger shift size. All triggers' positions are in a time window of $0.28 \mathrm{~ms}$. From this, it can be estimated that $t_{\text {overlap }} \leq 0.08 \mathrm{~ms}$. As the readout time $(6.4 \mathrm{~ms})$ of the freerunning camera is longer than the integration time $(2 \mathrm{~ms})$ plus the required buffer of $0.28 \mathrm{~ms}$, an interference-free operation is possible.

The second measurement series was conducted with varying parameters, such as different oscillator frequencies, investigating the synchronization error and the applicability of the synchronization procedure for real-world applications. Depending on real-world hardware and environmental conditions, the frequencies of the two cameras' oscillators will differ. To simulate different conditions, 1000 measurements were performed with different initial situations. For each measurement, the frequency difference and the time between the triggers of the free-running camera and the synchronizing camera were set to random values before the synchronization started. This was performed by substituting the cameras' oscillator signals with signals from a single programmable frequency generator providing two signals with user-defined frequency differences. The frequency differences ranged from 0 to $\pm 600 \mathrm{~Hz}$ ( $\pm 50 \mathrm{ppm})$ and the trigger's position in regards to the start of the time slot from 0 to $\pm 2.5 \mathrm{~ms}$. The synchronization interval was set to $1 \mathrm{~s}$. When the synchronized state was achieved, the trigger's position of the synchronizing camera is recorded using an oscilloscope. The necessary buffer is calculated using (2). With $t_{\text {overlap }}=0.08 \mathrm{~ms}$ from the first 


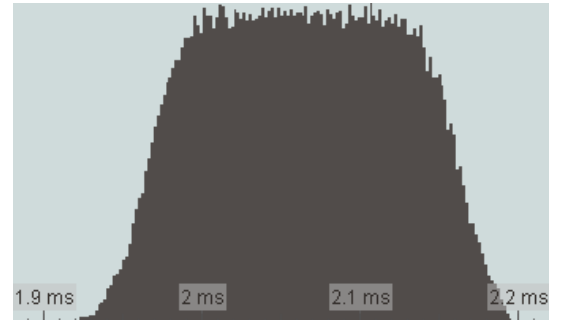

Fig. 7. Histogram screenshot showing the times between the triggers of the two independently running cameras. $99 \%$ fall into a time window of $0.24 \mathrm{~ms}$

TABLE I

TIME DIFFERENCE OF THE TWO TRIGGERS OF THE TWO INDEPENDENTLY RUNNING CAMERAS. CHARACTERISTIC VALUES OF THE HISTOGRAM

\begin{tabular}{|l|l|}
\hline Measure & Value \\
\hline Measurement time & $60 \mathrm{~min}$ \\
Count of measurements & $\approx 100000$ \\
Standard deviation $\sigma$ & $0.060 \mathrm{~ms}$ \\
Mean & $2.06 \mathrm{~ms}$ \\
Mean $\pm 2 \cdot \sigma$ & $99 \%$ \\
$2 \cdot 2 \cdot \sigma$ & $0.24 \mathrm{~ms}$ \\
Max - Min & $0.28 \mathrm{~ms}$ \\
\hline
\end{tabular}

measurement series and setting $t_{\text {drift }}=0.05 \mathrm{~ms}$ (maximum drift for the given conditions), the required buffer size is $t_{\text {buffer }}=2 \cdot(0.08 \mathrm{~ms})+\max (0.2 \mathrm{~ms}, 0.3 \mathrm{~ms})=0.46 \mathrm{~ms}$. TABLE II shows the statistics of the measurements. All of the triggers' positions could be shifted into a time interval of $0.31 \mathrm{~ms}$. The results demonstrate the synchronization procedure functions correctly for all expected frequency differences.

\section{DISCUSSION}

The measurement series support the theoretical proposition of an optical synchronizing algorithm. The results demonstrate that a ToF camera is able to detect another camera and determine the timing of the image acquisition. In addition, the control algorithm was able to shift the image acquisition trigger to stay synchronized. In the first measurement series, with two independently operating cameras, a synchronous operation throughout the measurement run was maintained. The difference between the measured and calculated buffer size is the anticipated overlap time $(0.08 \mathrm{~ms})$, which is scene dependent and appears relatively appropriate to the experimental conditions.

The second measurement series' measured time interval, accommodating all acquisition triggers' positions, is less than the calculated maximum buffer value. The reason for the interval to be smaller than the calculated buffer value might be that the initial positions of the acquisition trigger, in regards to the start of the time slots, were set randomly, and not all possible offsets were chosen during the 1000 measurement runs. As the calculated maximum buffer size was not exceeded during the measurements, the experiment proves the functionality of the synchronization procedure.

For the operation of two ToF cameras, the readout time is more than sufficient for the synchronous TDMA operation.
TABLE II

STATISTICAL MEASURES OF THE SYNCHRONIZATION FOR DIFFERENT INITIAL SCENARIOS

\begin{tabular}{|l|l|}
\hline Measure & Value \\
\hline Count of measurements & 1000 \\
Standard deviation $\sigma$ & $0.072 \mathrm{~ms}$ \\
Frequency difference & 0 to $\pm 50 \mathrm{ppm}( \pm 600 \mathrm{~Hz}$ at $12 \mathrm{MHz})$ \\
Mean $\pm 2 \cdot \sigma$ & $99 \%$ \\
Max - Min & $0.31 \mathrm{~ms}$ \\
\hline
\end{tabular}

The results indicate, in this setup, capacity for three operating ToF cameras including the buffer. Designing a system for more cameras necessitates further consideration of the control algorithm's parameters to shorten buffer times safely. Smaller synchronization intervals allow for smaller buffers compensating frequency drift. A reduction of the trigger shift size and gap size will reduce the synchronization error. These parameters can be modified to reduce the total buffer size allowing a greater number of operating cameras, but an absolute minimum is the overlap size (here $0.08 \mathrm{~ms}$ ).

Wübbenhorst conducted a similar experiment to synchronize two ToF cameras. Additional photodiodes were connected to the cameras in order to determine the exact timing of completion of the other camera's acquisition phase. His experiments demonstrated the completion of image acquisition was detected with an error of $4 \mu \mathrm{s}$ and a standard deviation of $560 \mathrm{~ns}$ [13]. Wübbenhorst's synchronization error is smaller than the required overlap time of $80 \mu \mathrm{s}$ of the proposed synchronization procedure. However, the paper's results were achieved with no additional hardware.

One requirement of the proposed synchronization procedure is the constant illumination time of the free-running camera. The fine-sync mode needs the constant illumination time in order to determine its trigger point in regards to the freerunning camera. With a variable illumination time of the freerunning camera, the synchronizing camera will not be able to precisely determine the synchronized state and therefore is impaired in determining the timing of the time slots. In normal camera operation the illumination time equals the integration time and therefore a constant integration time would be required. However, a constant integration time is not feasible in all practical applications. There are two possibilities which can fulfill both requirements, the variable integration time and the constant illumination time. The first one is to separate the illumination from the integration. That enables a varying integration time while the illumination can be active for a longer time. The second possibility is to introduce a dummy frame. This additional dummy frame is acquired before the actual frame. The integration time of the dummy is the difference of the required constant illumination time and the variable integration time of the actual frame. Both possibilities seem feasible but have not yet been implemented and tested. 


\section{CONCLUSION}

A multi-view time-of-flight camera operation is used to derive an accurate 3-D image of an environment. This work presents a new approach based on time division multiple access (TDMA) which avoids interference-related errors. A dynamic and expansive autonomous camera operation is achieved by overcoming the usual limitations of TDMA such as a static setup with wiring, the necessity of an external synchronizing device, or reduction of frame rates with an increasing number of sequential cameras. The cameras determine their time slots autonomously without any external synchronizing device or hard-wired communication hardware. The synchronization procedure is able to determine another camera's operational timing sequence and then adapt its image acquisition times by time-shifting its image acquisition trigger. The major contributing element is the cameras' self-management of their synchronization without any external control. The experimental results presented support the functionality of this approach. Therefore, dynamic environmental setups should be feasible where multiple robots with mounted $\mathrm{ToF}$ cameras may operate autonomously and adapt themselves to changing environments.

Extending the method and results to environments with more than two ToF cameras appears feasible. In this practical direction, the algorithms would need development for the detection of an increased number of active cameras. The limiting factors for a maximum number of ToF cameras operating without interference are the readout time of the ToF camera, the maximum integration time (depending on camera and scene), and the synchronization error. A reasonable hypothesis for the practical application of the promise of this research paper is to scale up setups to reach the required number of cameras to accurately and safely map a 3-D environment. Multiple clusters of cameras, using the proposed TDMA method within each cluster, can be configured utilizing a different access method between the clusters. Current multiToF-camera setups utilizing FDMA can reduce the required number of different frequencies with the clustering approach.

\section{REFERENCES}

[1] C. Savur, S. Kumar, S. Arora, T. Hazbar, and F. Sahin, "Hrc-sos: Human robot collaboration experimentation platform as system of systems," in 2019 14th Annual Conference System of Systems Engineering (SoSE), 05 2019, pp. 206-211.
[2] U. B. Himmelsbach, T. M. Wendt, and M. Lai, "Towards safe speed and separation monitoring in human-robot collaboration with 3d-timeof-flight cameras," in 2018 Second IEEE International Conference on Robotic Computing (IRC), Jan 2018, pp. 197-200.

[3] F. Flacco, T. Kroeger, A. D. Luca, and O. Khatib, "A depth space approach for evaluating distance to objects," Journal of Intelligent \& Robotic Systems, vol. 80, no. S1, pp. 7-22, oct 2014. [Online]. Available: https://doi.org/10.1007/s10846-014-0146-2

[4] S. Kumar, S. Arora, and F. Sahin, "Speed and separation monitoring using on-robot time-of-flight laser-ranging sensor arrays," in 2019 IEEE 15th International Conference on Automation Science and Engineering (CASE), Aug 2019, pp. 1684-1691.

[5] L. A. Seewald, V. F. Rodrigues, M. Ollenschläger, R. S. Antunes, C. A. da Costa, R. da Rosa Righi, L. G. da Silveira, A. Maier, B. Eskofier, and R. Fahrig, "Toward analyzing mutual interference on infrared-enabled depth cameras," Computer Vision and Image Understanding, vol. 178, pp. 1 - 15, 2019. [Online]. Available: http://www.sciencedirect.com/science/article/pii/S1077314218303400

[6] A. Kunz, L. Brogli, and A. Alavi, "Interference measurement of kinect for xbox one," in Proceedings of the 22Nd ACM Conference on Virtual Reality Software and Technology. ACM, 11 2016, pp. 345-346. [Online]. Available: http://doi.acm.org/10.1145/2993369.2996329

[7] S. Bauer, A. Seitel, H. G. Hofmann, T. Blum, J. Wasza, M. Balda, H.-P. Meinzer, N. Navab, J. Hornegger, and L. Maier-Hein, "Real-time range imaging in health care: A survey," in Time-of-Flight and Depth Imaging, 2013.

[8] J. Volak, D. Koniar, F. Jabloncik, L. Hargas, and S. Janisova, "Interference artifacts suppression in systems with multiple depth cameras," in 2019 42nd International Conference on Telecommunications and Signal Processing (TSP), 07 2019, pp. 472-476.

[9] Y. M. Kim, D. Chan, C. Theobalt, and S. Thrun, "Design and calibration of a multi-view tof sensor fusion system," in 2008 IEEE Computer Society Conference on Computer Vision and Pattern Recognition Workshops, June 2008, pp. 1-7.

[10] L. Li, S. Xiang, Y. Yang, and L. Yu, "Multi-camera interference cancellation of time-of-flight (tof) cameras," in 2015 IEEE International Conference on Image Processing (ICIP), Sep. 2015, pp. 556-560.

[11] B. Büttgen, Extending Time-of-flight Optical 3D-imaging to Extreme Operating Conditions. Sierke, 2007. [Online]. Available: https://books.google.de/books?id=eItHNAAACAAJ

[12] B. Buttgen and P. Seitz, "Robust optical time-of-flight range imaging based on smart pixel structures," IEEE Transactions on Circuits and Systems I: Regular Papers, vol. 55, no. 6, pp. 1512-1525, July 2008.

[13] T. Wübbenhorst, "Frame-basierte optische synchronisation von time-offlight (tof) sensoren," Master's thesis, Humboldt-Universität zu Berlin, 2019.

[14] R. Lange and P. Seitz, "Seeing distances - a fast time-of-flight 3d camera," Sensor Review, vol. 20, no. 3, pp. 212-217, 2000. [Online]. Available: https://doi.org/10.1108/02602280010372359

[15] S. Kim, O. Choi, B. Kang, J. Kim, and C. Y. Kim, "Time-of-flight depth image enhancement using variable integration time," Proceedings of SPIE - The International Society for Optical Engineering, vol. 8650, pp. 07-, 032013. 